\title{
An epigenetic cause of adaptive variation
}

Understanding the molecular basis of adaptive variation is one of the main goals of ecological and evolutionary genetics. Recent work from Shindo and colleagues reveals that variation in epigenetic silencing controls variation in flowering time in Arabidopsis thaliana - a key adaptive trait.

Arabidopsis thaliana plants need to be exposed to a period of cold before they can flower - a response called vernalization. The species grows in a wide range of environments, and plants that come from different environments show considerable differences in flowering times and vernalization responses. To understand the molecular basis of this adaptation to different environments, Shindo et al.

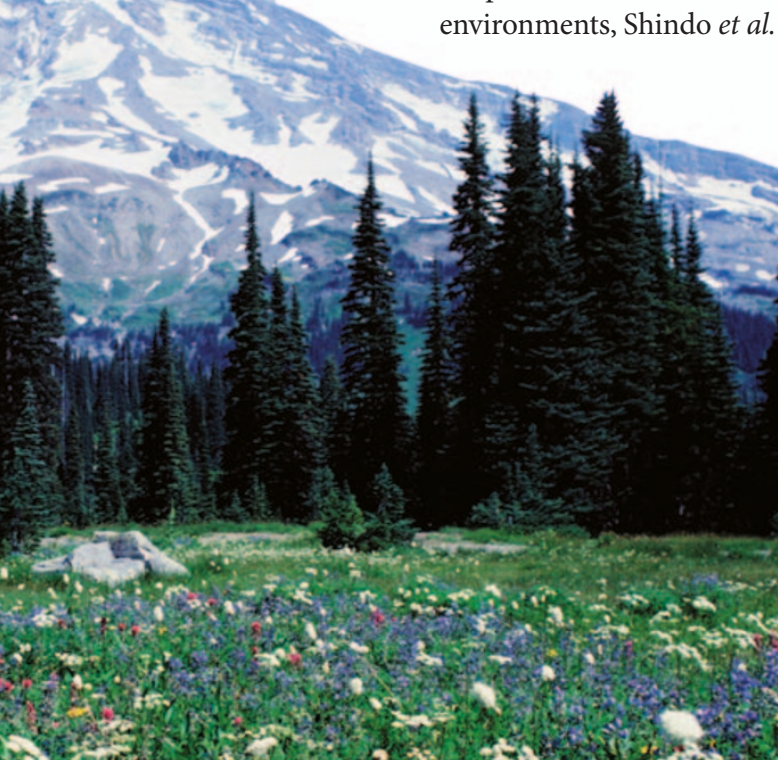

assessed expression levels of two markers: flowering locus $\mathrm{C}(F L C)$, the molecular target of vernalization, and vernalization insensitive 3 (VIN3), an early component of the response. To their surprise, there was no correlation between RNA levels of these two markers and the length of exposure to the cold.

Next, the authors looked at the stability of FLC repression - when expressed, FLC represses flowering; vernalization alleviates this effect by repressing FLC expression through an epigenetic mechanism. In this case they found a difference - in plants that respond more slowly to vernalization (collected from northern locations), FLC RNA levels increased significantly once the period of cold was over, whereas in plants with faster responses to vernalization (collected from more southern locations) these RNA levels essentially did not change. In fact, the authors found that increasing the length of the cold period quantitatively enhanced the stability of FLC repression. Chromatin immunoprecipitation (ChIP) experiments showed that, in molecular terms, variation in the response to vernalization translates into differential accumulation of histone $\mathrm{H} 3$ lysine 27 methylation.

QTL analysis in four A. thaliana populations revealed that genetic determinants of the variation in vernalization response mapped to chromosomes 1, 4 and 5, distributed across four or five distinct loci. Notably, however, these loci do not correspond to any of the three known trans factors (VRN1, VRN2 and VIN3) that are thought to directly affect the stability of FLC silencing. The mapping data also indicate that the FLC locus itself might contribute to the variation. Although the $5^{\prime}$ region and the first intron of FLC have been shown to be important for the maintenance of FLC repression, key cis elements remain to be identified.

On the basis of the ChIP data, flowering time and FLC expression analysis, the authors propose that vernalization occurs in three phases: an insensitive phase is followed by a phase in which silencing occurs; when the levels of histone modification are sufficient, the third phase begins, in which FLC is fully silenced. The time taken to accumulate sufficient histone methylation levels determines the speed of the response to vernalization. The hunt for the genes that underlie the identified QTLs and that control this natural variation in vernalization response is on.

Magdalena Skipper

ORIGINAL RESEARCH PAPER Shindo, C. et al. Variation in the epigenetic silencing of FLC contributes to natural variation in Arabidopsis vernalization response. Genes Dev. 20 , 3079-3083 (2006)

FUTHER READING Orr, H. A. The genetic theory of adaptation: a brief history. Nature Rev. Genet. 6 , 119-127 (2005)

\section{RESEARCH HIGHLIGHTS ADVISORS}

MICHAEL AKAM

University of Cambridge, UK SEAN B. CARROLL

University of Wisconsin, USA NANCY J. COX

University of Chicago, USA

SUSAN FORSBURG University of

Southern California, USA
RALPH J. GREENSPAN

The Neurosciences Institute, California, USA YOSHIHIDE HAYASHIZAK Riken Genomic Sciences Center, Japan MARK JOBLING

University of Leicester, UK
PETER KOOPMAN

University of Queensland, Australi LEONID KRUGLYAK Fred Hutchinson Cancer Research Center, USA BARBARA MEYER

University of California, Berkeley, USA
JOHN QUAKENBUSH

Dana-Farber Cancer Institute and Harvard School of Public Health, Boston, USA JANET ROSSANT

Mount Sinai Hospital, Toronto, Canad MARC VIDAL Dana-Farber Cancer Institute, Boston, USA
VIRGINIA WALBOT

Stanford University, USA

DETLEF WEIGEL Max Planck Institute for Developmental Biology, Germany PHIL ZAMORE

University of Massachusetts, USA LEONARD I. ZON

Children's Hospital, Boston, USA 